\title{
Spin orientation of supermassive black holes in active galaxies $^{\star}, \star \star$
}

\author{
W. Kollatschny ${ }^{\star \star \star}$ \\ Universitäts-Sternwarte Göttingen, Geismarlandstraße 11, 37083 Göttingen, Germany \\ Received 14 October 2003 / Accepted 10 November 2003
}

\begin{abstract}
Accretion of gas onto a central supermassive black hole is generally accepted to be the source of the emitted energy in active galactic nuclei. The broad emission lines we observe in their optical spectra are probably formed in the wind of an accretion disk at distances of light days to light years from the central black hole. The variable fraction of the emission lines originates at typical distances of only 1 to 50 light days from the central supermassive black hole. We derived a central black hole mass of $M_{\text {orbital }}=1.8 \pm 0.4 \times 10^{7} M_{\odot}$ in the Seyfert galaxy Mrk 110 assuming the broad emission lines are generated in gas clouds orbiting within an accretion disk. This figure depends on the inclination angle of the accretion disk. Here we report on the detection of gravitational redshifted emission in the variable fraction of the broad emission lines. We derive a central black hole mass of $M_{\text {grav }}=14.0 \pm 3.0 \times 10^{7} M_{\odot}$. These measurements are independent on the orientation of the accretion disk. The comparison of both black hole mass estimates allows to determine the projection of the central accretion disk angle $i$ to $21 \pm 5 \mathrm{deg}$ in Mrk 110 and therefore the orientation of the spin axis of the central black hole.
\end{abstract}

Key words. accretion disks - black hole physics - gravitation - line: profiles - galaxies: active galaxies: individual: Mrk 110

\section{Introduction}

Mass and spin of the central black holes in galaxies as well as geometry and kinematics of their immediate stellar and gaseous surroundings are of fundamental interest for understanding the origin of the enormous energies emitted in the nuclear regions of active galaxies (e.g. Blandford \& Begelman 1999; Murray $\&$ Cheng 1997). Furthermore, the central black hole masses and their evolution might be connected to the general properties of their host galaxies. The black hole mass has been estimated in some active galaxies by reverberation mapping (e.g. Wandel et al. 1999). A basic assumption of this method is that the broad emission line gas is gravitationally dominated by the central black hole. Here a major uncertainty is introduced by the geometry and orientation of the line-emitting gas. An independent mass determination of the central black hole can be obtained by the gravitational redshift effect in the optical emission lines of active galaxies. However, in this respect only the detection of a gravitationally redshifted $\mathrm{Fe} \mathrm{K} \alpha \mathrm{X}$-ray line has been reported in a few active galaxies (Tanaka et al. 1995; Fabian et al. 2000).

* Based on observations obtained with the Hobby-Eberly Telescope, which is a joint project of the University of Texas at Austin, the Pennsylvania State University, Stanford University, Ludwig-Maximilians-Universität München, and Georg-AugustUniversität Göttingen.

$\star \star$ This paper is dedicated to Frank Bash without whose efforts the Hobby-Eberly Telescope would not have been possible.

$\star \star \star$ e-mail: wkollat@uni-sw.gwdg.de

\section{Observations and data reduction}

We selected the narrow-line Seyfert 1 galaxy Mrk 110 as our primary target to determine the central black hole mass independently both by reverberation mapping and by gravitational redshift effects. From earlier long term campaigns we knew of the extreme continuum and line intensity variations in this galaxy (Bischoff \& Kollatschny 1999; Peterson et al. 1998). We carried out our optical variability campaign with the $9.2 \mathrm{~m}$ Hobby-Eberly Telescope (HET) at McDonald Observatory. We took 26 spectra of Mrk 110 between 1999 November 13 and 2000 May 14. All observations were made under identical conditions with exactly the same instrumentation at the HET. The spectra cover a wavelength range from $4200 \AA$ to $6900 \AA$ with a resolving power of 650 . The data were reduced in a homogeneous way with IRAF reduction packages. In most cases we reached a $S / N>100$ per pixel in the continuum. Great care was taken of a very accurate internal calibration of the spectra with respect to wavelength and flux (Kollatschny et al. 2001).

\section{Results and discussion}

\subsection{Mean and rms line profiles}

We determined mean and rms emission line profiles of the most intense lines $\mathrm{H} \alpha, \mathrm{H} \beta, \mathrm{HeI} \lambda 5876$, and HeII $\lambda 4686$ from our variability campaign. The rms profiles are a measure of the variable component of the line profile. The profiles shown in Fig. 1 are plotted in velocity space and normalized to the same maximum intensity. The fraction of the variable component in these lines 

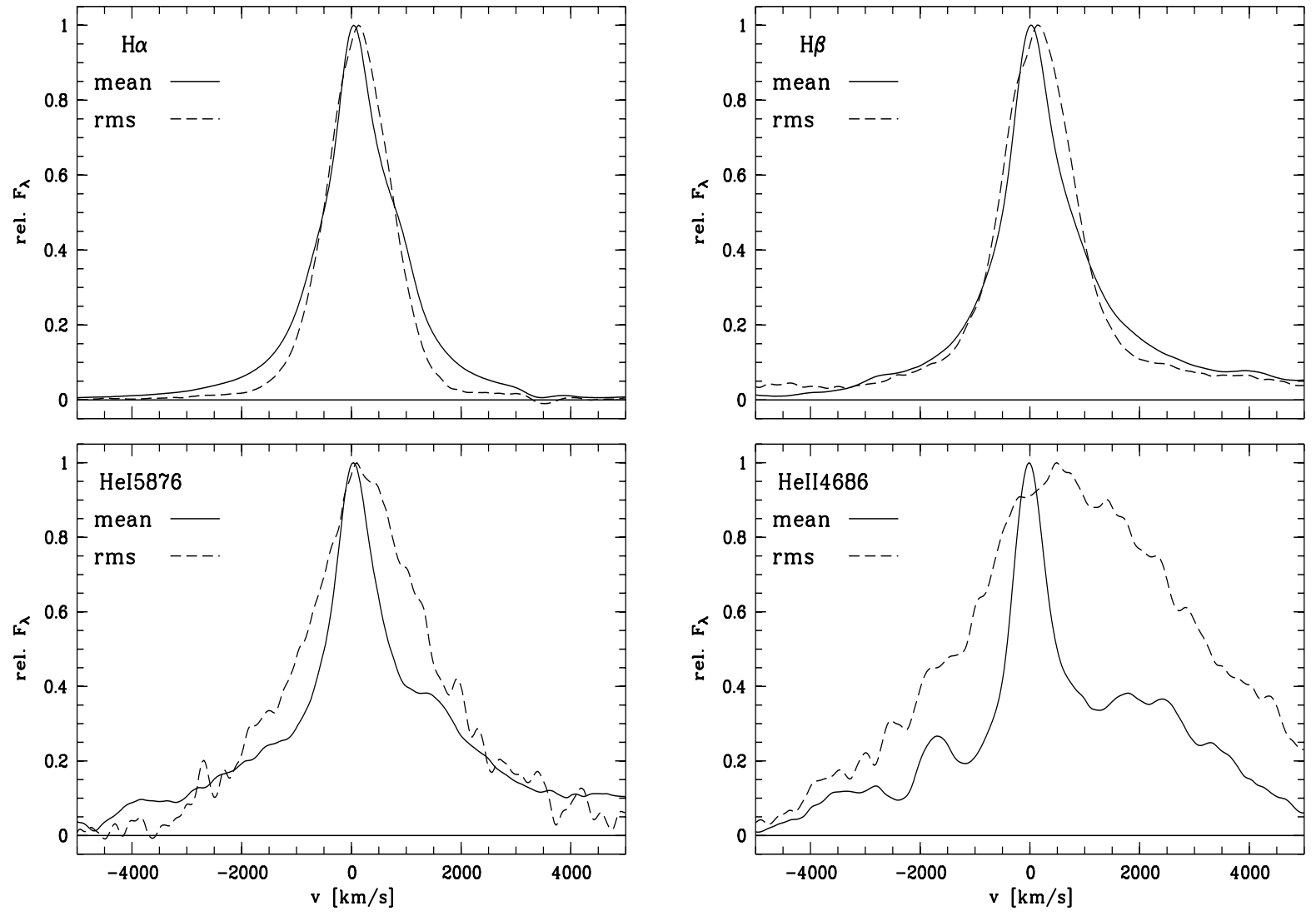

Fig. 1. Normalized mean (dashed lines) and rms (solid lines) Balmer and helium emission line profiles.

Table 1. Rms line widths $(F W H M)$ of our strongest emission lines: $\mathrm{H} \alpha, \mathrm{H} \beta, \mathrm{HeI} \lambda 5876$, and HeII $\lambda 4686$; differential redshift of rms line centers $\Delta v_{\text {cent }}(\mathrm{rms})$; cross-correlation lags $\tau$ and central black hole mass estimation $M_{\text {grav }}$ derived from gravitational redshift.

\begin{tabular}{|c|c|c|c|c|}
\hline Line & $\begin{array}{c}F W H M(\mathrm{rms}) \\
{\left[\mathrm{km} \mathrm{s}^{-1}\right]} \\
(2)\end{array}$ & $\begin{array}{c}\Delta v_{\text {cent }}(\mathrm{rms}) \\
{\left[\mathrm{km} \mathrm{s}^{-1}\right]} \\
(3)\end{array}$ & $\begin{array}{c}\tau \\
\text { [days] } \\
(4)\end{array}$ & $\begin{array}{c}M_{\text {grav }} \\
{\left[10^{7} M_{\odot}\right]} \\
(5)\end{array}$ \\
\hline HeII & $4444 \pm 200$ & $541 \pm 60$ & $3.9 \pm 2$ & $13 \pm 3$ \\
\hline $\mathrm{HeI}$ & $2404 \pm 100$ & $186 \pm 60$ & $10.7 \pm 6$ & $12 \pm 4$ \\
\hline $\mathrm{H} \beta$ & $1515 \pm 100$ & $118 \pm 50$ & $24.2 \pm 4$ & $17 \pm 4$ \\
\hline $\mathrm{H} \alpha$ & $1315 \pm 100$ & $74 \pm 50$ & $32.3 \pm 5$ & $14 \pm 5$ \\
\hline
\end{tabular}

amounts to $9,12,14$, and $39 \%$, respectively. The line widths $(F W H M)$ of the rms profiles are listed in Table 1.

\subsection{Virial mass of the central black hole}

We calculated the characteristic distances of the line emitting regions by cross-correlating the light curves of the emission lines with the optical continuum light curve. The broader emission lines originate systematically closer to the galaxy center in Mrk 110 (see Table 1) (Kollatschny et al. 2001; Kollatschny 2003). The fraction of the variable line component is correlated with the distance of the line emitting region from the black hole. The distances of the line emitting regions we determined from the cross-correlation function $(\mathrm{CCF})$ analysis correspond to the widths of the rms profiles (Table 1). The non-variable components of the emission lines originate at larger distances from the black hole. We determined the central black hole mass assuming Keplerian orbits of the line emitting clouds:

$M_{\text {orbital }}=f v^{2} G^{-1} R$.

where $v$ is the characteristic emission-line velocity (rms line width), $G$ is the gravitational constant, and $R=\tau c$ is the distance of the line emitting region $(\tau=$ calculated crosscorrelation lag of line emitting region). The derived black hole mass in Mrk 110 is

$M_{\text {orbital }}=1.8 \pm 0.4 \times 10^{7} M_{\odot}$

The dimensionless factor $f$ depends amongst other things on the geometry and (unknown) orientation of the BLR. We could show that the velocities in the broad emission line region are not randomly oriented as for instance in a turbulent flow but that the lines are formed in an accretion disk (Kollatschny et al. 2002; Kollatschny 2003). The line widths of the rms spectral lines are interpreted as the line-of-sight Doppler widths. We observe only the projected velocity $v \sin i$ of the rotating accretion disk. Thus, the derived mass is a lower limit. The systematic errors in the estimation of black hole masses by reverberation mapping have been discussed by Krolik (2001). He pointed at a systematic underestimate of the central AGN mass caused by the possible inclination of the line emitting region. 


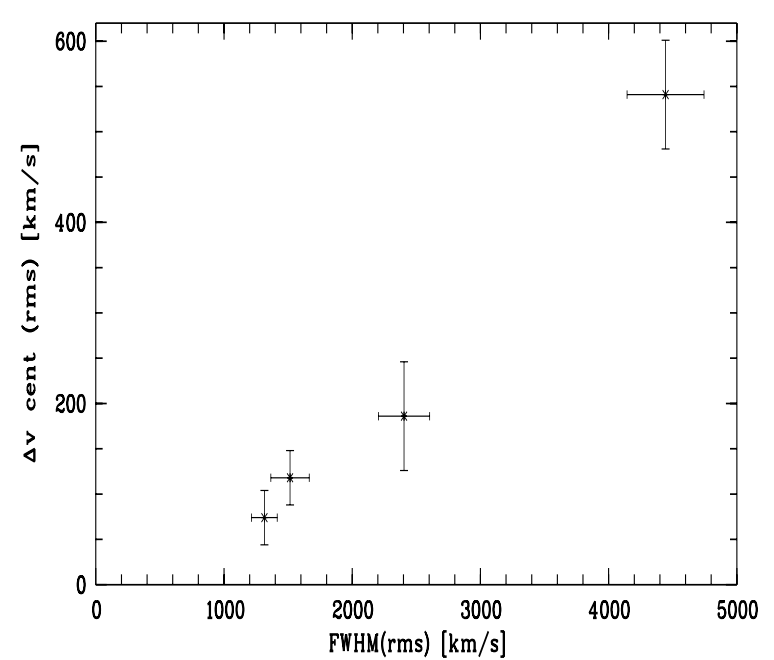

Fig. 2. Relative redshift of $\mathrm{H} \alpha, \mathrm{H} \beta$, HeI $\lambda 5876$, and HeII $\lambda 4686 \mathrm{rms}$ line centers as a function of rms line width ( $F W H M$ ) (see Table 1).

\subsection{Emission line shifts}

We determined from the spectra in Fig. 1 the shift of the rms line centers (uppermost 20\%). It is a known effect that rms line profiles sometimes show narrow forbidden line residuals that are caused by variable seeing and small shifts in wavelength calibration (e.g. Kaspi et al. 2000). The degree of this contamination is strongest in the HeI $\lambda 5876$ line which is the weakest one. The redshift $(\Delta v)$ of the rms profiles with respect to the narrow emission lines increases as a function of line width and ionization potential as seen in Fig. 1. The correlation of rms line width with observed central line shift is shown in Fig. 2.

High-ionization broad emission lines originate closer to the central supermassive black hole than the low-ionization ones (e.g. Peterson \& Wandel 1999; Kollatschny et al. 2001). The redshifts derived from different integrated emission lines in AGN often do not agree with each other. High-ionization broad emission lines are sometimes blueshifted with respect to the low-ionization broad emission lines and narrow emission lines (Gaskell 1982; Sulentic et al. 2000). Therefore, the shift of the integrated broad line profiles cannot be explained by gravitationally redshift effects. But is has been demonstrated recently that the observed blueshift in the highly ionized lines is mimicked by absorption in the red line wings (Richards et al. 2002). This effect is more pronounced in the higher ionized lines. Additionally, one has to consider that the integrated emission lines originate at distances of light weeks to light years from the black hole. A verification of a gravitational redshift in these lines is beyond the detection limit.

\subsection{Central black hole mass derived from gravitational redshift}

The variable broad line components originate far more closely to the central black hole than the constant line components. A gravitational redshift effect in this variable component might thus be present. We measured the differential shifts $\Delta v=\Delta z c$ of the strongest rms emission profiles with respect to their mean line profiles (Fig. 1, Table 1). The observed shifts of the

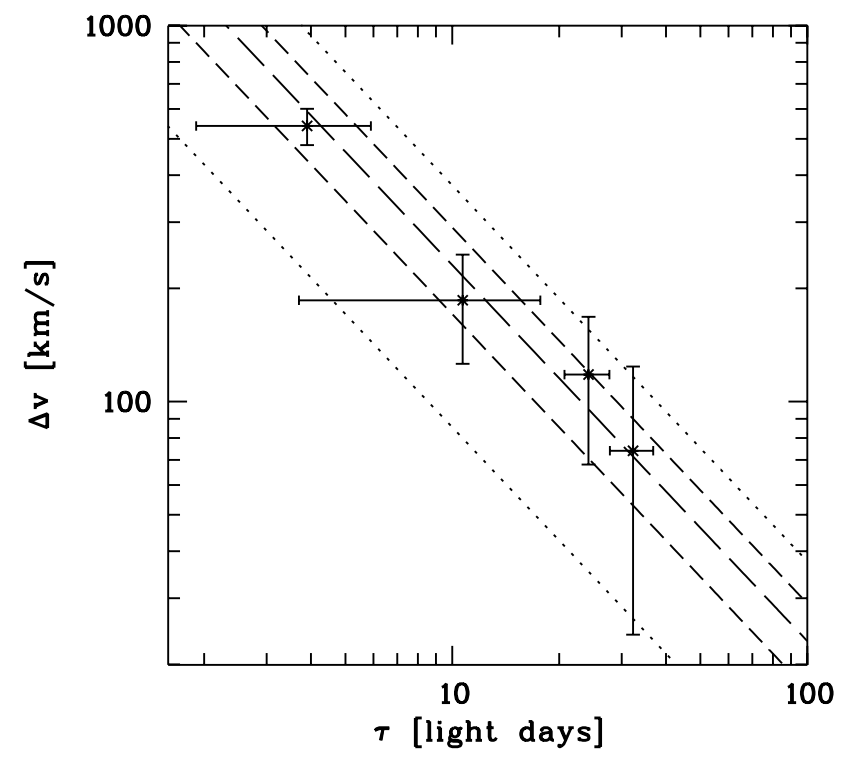

Fig. 3. Redshift of the rms profiles of the HeII $\lambda 4686$, HeI $\lambda 5876$, $\mathrm{H} \beta$ and $\mathrm{H} \alpha$ lines as a function of the distance of the rms line emitting region (see Table 1). The dotted and dashed curves show computed lines of gravitational redshifts for central masses of 5.0, 10.0, 13.5, 17.0, $22.0 \times 10^{7} M_{\odot}$ (from bottom to top).

rms profiles are identified as gravitational redshifts. The mean profiles of the strongest broad emission lines are not shifted with respect to the forbidden narrow lines on the other hand. Therefore, the differential shifts of the rms profiles with respect to the narrow lines are identical to their shifts with respect of the mean profiles.

We calculated the central black hole mass $M_{\text {grav }}$ in Mrk 110 using the formula (e.g. Zheng \& Sulentic 1990)

$M_{\text {grav }}=c^{2} G^{-1} R \Delta z$.

The distances $R(R=c \tau)$ of the individual line emitting regions are known from the cross-correlation analysis (Table 1). We determined a black hole mass $M_{\text {grav }}$ of $12 \pm 4$ to $17 \pm 4 \times 10^{7} M_{\odot}$ with a mean value of

$M_{\text {grav }}=14 \pm 3 \times 10^{7} M_{\odot}$.

from the line shifts. The rms line profile shifts are shown in Fig. 3 as a function of distance to the center.

The internal shifts of all four emission lines $(\mathrm{H} \alpha, \mathrm{H} \beta$, HeI 25876 , and HeII $\lambda 4686$ ) are different. They depend on the distance of the line emitting region from the center. It is intriguing that the black hole masses we derived from the four different line shifts are identical within the error limits. This verifies our interpretation of the observed differential line shifts as gravitaional redshifts.

The dotted and dashed lines represent theoretical models. They show the correlation between distance (i.e. crosscorrelation lag $\tau$ ) and gravitational redshift $\Delta v$ for different central black hole masses $M_{\text {grav }}$ of 5.0, 10.0, 13.5, 17.0, and $22.0 \times 10^{7} M_{\odot}$, respectively.

Other evidence for the detection of a gravitational redshift has been shown before in the spectra of the Seyfert galaxy NGC 4593 (Kollatschny et al. 1997). In this galaxy the 
Balmer lines originate at distances of 2 to 4 light days only. On the other hand, the quality of the spectra in the NGC 4593 variability campaign was not as good as in Mrk 110. The derived black hole mass $M_{\text {grav }}=2.3 \times 10^{7} M_{\odot}$ in NGC 4593 was by a factor of 1.6 higher in comparison to the mass $M_{\text {orbital }}$ derived from the line widths. An additional hint for a gravitational redshift can be seen in the HST spectra of the NGC 5548 variability campaign. The line center of the rms CIV $\lambda 1550$ profile is redshifted with respect to the mean profile by about $10 \AA$ (see Fig. 4 in Peterson 2001).

\subsection{Inclination angle of central accretion disk}

The determination of black hole masses in AGNs through their gravitational redshift effect has clear advantages. The derived central black hole mass $M_{\text {grav }}$ is not affected by the orientation of the central accretion disk in contrast to the mass $M_{\text {orbital }}$ derived from the emission line widths. It has been mentioned before that only an accretion disk model of the BLR in Mrk 110 can reproduce the observed line profile variations (Kollatschny et al. 2001; Kollatschny 2003). The mutual comparison of both mass estimates gives us information on the orientation angle $i$ of the accretion disk: $M_{\text {orbital }} / M_{\text {grav }}=\sin ^{2} i$. For the narrow line Seyfert galaxy Mrk 110 we derive a nearly pole-on line-of-sight of the central accretion disk. The inclination angle $i$ amounts to $21 \pm 5 \mathrm{deg}$. A disk inclination angle of $30 \pm 20 \mathrm{deg}$ has been previously estimated from line profile variations (Kollatschny 2003). This confirms independently the nearly pole-on view of the accretion disk in Mrk 110.

The variable HeII line component originates at a mean distance of 3.9 light-days $\left(\stackrel{\wedge}{=} 9.8 \times 10^{15} \mathrm{~cm}\right)$ only. This distance corresponds to 230 Schwarzschild radii $r_{\mathrm{s}}\left(r_{\mathrm{s}}=2 G M_{\text {grav }} / c^{2}\right)$ for a central black hole mass of $14 \pm 3 \times 10^{7} M_{\odot}$. It is very plausible that the rotation axis of the inner accretion disk is oriented parallel to the spin axis of the central black hole. Altogether, the black hole mass $M_{\text {grav }}$ derived from the gravitational redshift is not only more reliable: a comparison with the black hole mass $M_{\text {orbital }}$ deduced from the line widths opens up the possibility to determine the orientation of the central black holes in radio-quiet AGN. A good test of the method proposed here would be to check the inclination of optical broad emission line regions with that of radio/optical jets in radio-loud objects.

Acknowledgements. WK thanks the UT Astronomy Department for warm hospitality during his visit. He thanks K. Bischoff and M. Zetzl for valuable comments. Part of this work has been supported by the Deutsche Forschungsgemeinschaft, DFG grant KO 857/24 and DAAD.

\section{References}

Bischoff, K., \& Kollatschny, W. 1999, A\&A, 345, 49

Blandford, R. D., \& Begelman, M. C. 1999, MNRAS, 303, L1

Fabian, A. C., Iwasawa, K., Reynolds, C. S., \& Young, A. J. 2000, PASP, 112, 1145

Gaskell, M. 1982, ApJ, 263, 79

Kaspi, S., Smith, P. S., Netzer, H., et al. 2000, ApJ, 533, 631

Kollatschny, W. 2003, A\&A, 407, 461

Kollatschny, W., \& Bischoff, K. 2002, A\&A, 386, L19

Kollatschny, W., \& Dietrich, M. 1997, A\&A, 323, 5

Kollatschny, W., Bischoff, K., Robinson, E. L., Welsh, W. F., \& Hill, G. J. 2001, A\&A, 379, 125

Krolik, J. H. 2001, ApJ, 551, 72

Murray, N., \& Cheng, J. 1997, ApJ, 474, 91

Peterson, B. M 2001, Proc. of a Conf. on The starburst-AGN connection, ed. I. Aretxaga (Singapore: World Scientific), 3

Peterson, B. M., Wanders, I., Bertram, R., et al. 1998, ApJ, 501, 82

Peterson, B. M., \& Wandel, A. 1999, ApJ, 521, L95

Richards, G. T., Vanden Berk, D. E., Reichard, T. A., et al. 2002, AJ, 124,1

Sulentic, J. W., Marziani, P., \& Dultzin-Hacayan, D. 2000, ARA\&A, 38,521

Tanaka, Y., Nandra, K., Fabian, A. C., et al. 1995, Nature, 375, 659

Wandel, A., Peterson, B. M., \& Malkan, M. A. 1999, ApJ, 526, 579

Zheng, W., \& Sulentic, J. W. 1990, ApJ, 350, 512 